\title{
THE SPIN-VALVE TRANSISTOR - A NEW MAGNETOELECTRONIC DEVICE
}

\author{
P.S. ANIL Kumar and J.C. LODder \\ Information Storage Technology Group, MESA+ Research Institute \\ University of Twente, 7500 AE Enschede, The Netherlands
}

A spin-valve transistor showing high sensitivity at low fields was developed. A large magnetocurrent, above $500 \%$ is realized by a magnetic field change of 2 to 4 Oe at $80 \mathrm{~K}$. Hot electrons are injected into the spin-valve layer through a Si-Pt Schottky diode. These hot electrons, while traversing through the spin-valve, are spin-dependently scattered. Those electrons with right energy and momentum are collected by a collector (an Au-Si Schottky diode) constituting a collector current. The relative orientation of the magnetic layer in the spin-valve is changed by the application of a magnetic field and causes a change in collector current giving a large magnetocurrent.

PACS numbers: 75.70.-i, 75.70.Pa

\section{Introduction}

Ever since the discovery of giant magnetoresistance (GMR) in metallic multilayers [1], the search for new devices based on GMR is being pursued in accordance with the demands arising from technological applications. The potential use of these devices [2] are as magnetic read heads and nonvolatile memory applications viz. magnetic random access memories (MRAM). The main parameters of importance from the read head point of view is high sensitivity for low applied magnetic fields. In MRAM, in addition to this a well-defined switching of the magnetic layers is required.

The GMR or the spin-valve effect is realized in metallic multilayer where alternating layers of ferromagnetic and non-magnetic metallic layers are stacked together. In such a multilayer when the charge carriers viz. the electrons are passed through and if the magnetic moments of the ferromagnetic layers are aligned in one direction, the spin-dependent scattering of the electrons is smaller and it results in lower electrical resistance for the multilayer. On the other hand, if the ferromagnetic layers are aligned opposite to each other, the spin-dependent scattering for the electrons is high and it results in higher electrical resistance. The relative orientation of the ferromagnetic layers can be intelligently controlled by the suitable combination of the ferromagnetic layers as well as the thickness of the non-magnetic spacer layer. This alignment can be controlled by an applied 
magnetic field and in turn the resistance of the multilayer is sensitive to magnetic field.

The conventional GMR materials show a large effect $\approx 65 \%$ at room temperature but high field is required $(\approx 10 \mathrm{kOe})$ to obtain this effect [3]. The GMR phenomena have been characterized with two different experimental configurations, namely the current in plane (CIP) and current perpendicular to the plane (CPP). In CIP mode the magnetoresistance is diminished by shunting or channeling by non-magnetic spacer layer. It has been showed that the CPP configuration gives better sensitivity towards magnetic field as the charge carriers pass through all the magnetic layers and it gives larger effect compared to the CIP configuration [4]. However, the low resistance across the multilayer stack in the CPP geometry offers experimental difficulty. Another structure was also proposed and demonstrated where a low coercive ferromagnetic layer is separated from the high coercive second ferromagnetic layer by a metallic spacer layer [5]. Each of this ferromagnetic layer can be switched independently by applying suitable magnetic fields. A magnetoresistance of $\approx 14 \%$ at room temperature has been observed in such kind of structures at low fields [6]. Another breakthrough is the demonstration of large magnetoresistance at room temperature using tunnel junctions where two
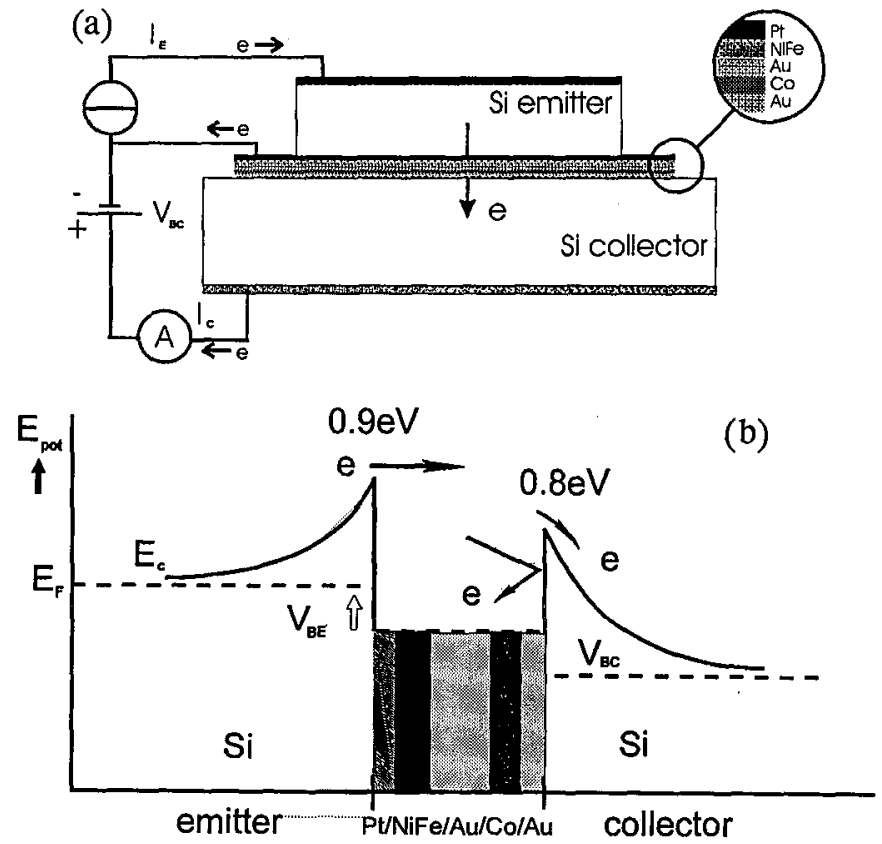

Fig. 1. (a) The schematic cross-section of the spin-valve transistor. The emitter is forward biased and the collector is reverse biased. $I_{E}$ is emitter current and $I_{C}$ is the collector current. The spin-valve layer in the base is also shown. (b) The schematic energy diagram of the spin-valve transistor. The Schottky barrier height for $\mathrm{Si}-\mathrm{Pt}$ and $\mathrm{Si}-\mathrm{Au}$ diode is shown. $V_{\mathrm{BC}}$ is the collector bias and $V_{\mathrm{BE}}$ is the emitter bias. 
ferromagnetic layers were separated by an insulating layer. A magnetoresistance of $11.8 \%$ at room temperature was observed at low fields [7].

In this search for new devices, a new magnetoelectronic device based on hot-electron transport - the spin-valve transistor - was invented in our lab [8]. The spin-valve transistor is analogous to a metal base transistor where the metal base is replaced by a spin-valve layer (permalloy $(\mathrm{Py}) / \mathrm{Au} / \mathrm{Co}$ ). In Fig. 1a, the schematic cross-section of the spin-valve transistor is given. A Pt layer is incorporated on one side of the spin-valve layer and $\mathrm{Au}$ is incorporated on the other side to form the Schottky barriers. The emitter diode is forward biased and the collector diode is reverse biased. In Fig. $1 \mathrm{~b}$ the schematic energy diagram of the spin-valve transistor is given. The emitter diode has a barrier height of nearly $0.85 \mathrm{eV}$ and the collector diode nearly $0.75 \mathrm{eV}$. A forward biasing of the emitter injects hot electrons into the spin-valve layer. These hot electrons, while traversing through the spin-valve layer, undergo elastic as well as inelastic scattering. Those electrons with energy greater than the collector barrier height can pass across the collector barrier constituting a collector current. Another factor which determines the ability of the electron to pass across the collector barrier is its momentum. The electrons which arrive at the collector barrier with an angle very close to its normal are only allowed to pass across [9]. Therefore the collector current strongly depends on the scattering of electrons in the base layer. The spin-dependent scattering of the electrons is believed to have a great influence on the collector current.

In this article the development of a spin-valve transistor as shown in Fig. 1 is presented.

\section{Experimental}

\subsection{Vacuum metal bonding}

The difficulty in growing device quality crystalline Si on top of metal layers has prompted us to use a vacuum metal bonding technology [10]. A bonding tool is used to bond two $\mathrm{Si}$ wafers together with a metal layer in between them. At first a thermal oxide is grown on top of the $\mathrm{Si}$ wafer to move the polishing damages. The wafer is annealed at high temperature to obtain a well-defined $\mathrm{Si} / \mathrm{SiO}_{2}$ interface. A protective photoresistive layer is coated on this wafer. The wafer is then sawn into small pieces to fit into the arms of the bonding tool. These small pieces were first cleaned to remove the protective resist layer. The sawdust is then removed and finally the thermally grown oxide layer is removed to obtain clean Si surfaces. These pieces were carefully transferred to the bonding tool. The bonding tool is then transferred to a molecular beam epitaxy (MBE) system. Metal layer is then grown on these Si wafers and finally the bonding tool brings the two Si wafers with metal layer on top of it in contact with each other in vacuum so as to obtain a bonded structure. In order to understand the efficiency of such a bonding method we have tested the bonding of different metal layers between Si wafers [10]. Figure 2 shows the cross-sectional transmission electron microscopic (TEM) image of an $\mathrm{Au}$ bond. Au layer of $5 \mathrm{~nm}$ is first grown on both Si wafers and then bonded together. It is clearly seen from the figure that this method gives a high quality bonding and an interface between the two Au layers is not visible. In case of the spin-valve transistor the spin-valve layer is grown on one of the wafers while a shutter masks 


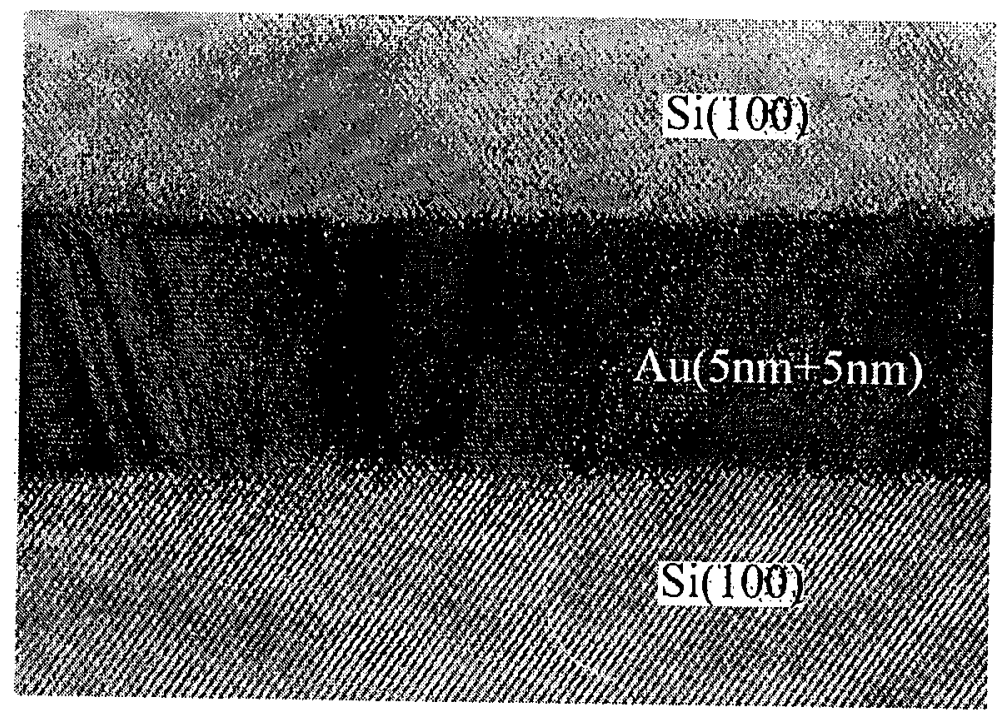

Fig. 2. The cross-sectional TEM of $\mathrm{Si} / \mathrm{Au}(5 \mathrm{~nm})-\mathrm{Au}(5 \mathrm{~nm}) / \mathrm{Si}$ bonded structure.

the second wafer. After the growth of the spin-valve layer the shutter is opened and $\mathrm{Au}$ is deposited on both the wafers and the bonding is carried out.

\subsection{Optimization of spin-valve layer}

At first a Pt layer of $2 \mathrm{~nm}$ is grown directly on clean $\mathrm{Si}(100)$ wafer so as to create a high Schotkky barrier on the emitter side. The spin-valve layer is then grown on this $\mathrm{Pt}$ layer. It is found that the growth of $3 \mathrm{~nm}$ of $\mathrm{Py}\left(\mathrm{Ni}_{0.81} \mathrm{Fe}_{0.19}\right)$ followed by $3.5 \mathrm{~nm}$ of $\mathrm{Au}$ and $3 \mathrm{~nm}$ of Co gives a well-defined switching of the magnetic layers. In Fig. 3 the magnetic field dependence of magnetization of such

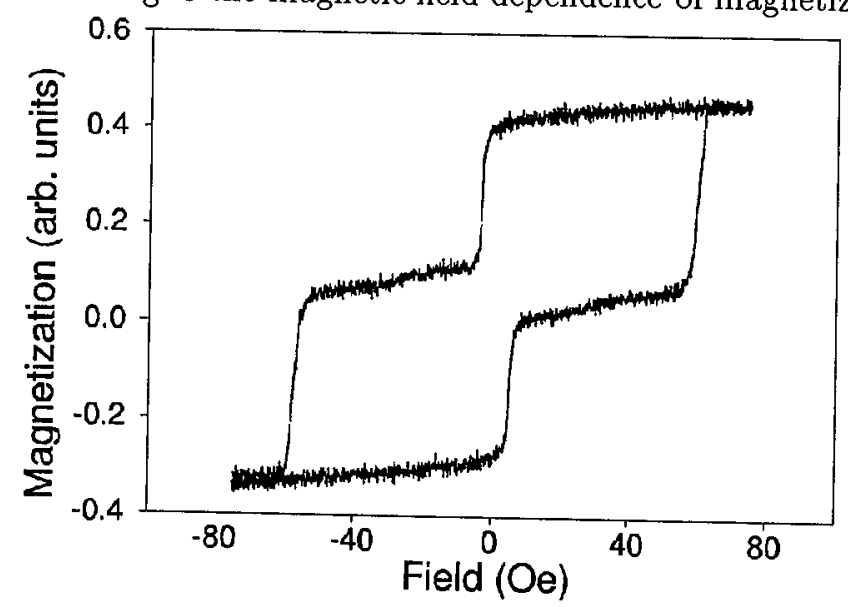

Fig. 3. The magnetic field dependence of the magnetization of $\mathrm{Si} / \operatorname{Pt}(2 \mathrm{~nm}) /$ $\mathrm{Py}(3 \mathrm{~nm}) / \mathrm{Au}(3.5 \mathrm{~nm}) / \mathrm{Co}(3 \mathrm{~nm})$ sample. 
an optimized spin-valve layer is given. It is clearly seen that the Py and the Co layers switch independently at the respective coercive fields indicating a good quality spin-valve layer.

\subsection{Fabrication of spin-valve transistor}

At the emitter and collector side of the spin-valve transistor, $n$-type device quality $\mathrm{Si}(100)$ and $\mathrm{Si}(111)$ wafers were used. Then the Pt layer is grown on $\mathrm{Si}(100)$ followed by the spin-valve layer. Subsequently, Au layer of $2 \mathrm{~nm}$ is grown on both $\mathrm{Si}(100)$ and $\mathrm{Si}(111)$ then the bonding is carried out between the Au layers to obtain the structure $\mathrm{Si}(100) / \mathrm{Pt}(2 \mathrm{~nm}) / \mathrm{NiFe}(3 \mathrm{~nm}) / \mathrm{Au}(3.5 \mathrm{~nm}) / \mathrm{Co}(3 \mathrm{~nm}) / \mathrm{Au}(2+2 \mathrm{~nm}) /$ $\mathrm{Si}(111)$. The $\mathrm{Si}(100)$ at the emitter side is thinned down to $30 \mu \mathrm{m}$ using $10 \%$ TMAH at $80^{\circ} \mathrm{C}$. Now $350 \times 350 \mu \mathrm{m}^{2}$ emitter is defined using photolithography and the remaining $\mathrm{Si}$ is etched away using TMAH. Now $700 \times 350 \mu \mathrm{m}^{2}$ base layer is defined and the remaining metal layer was etched away using dry iron beam etching. This spin-valve transistor with $350 \times 350 \mu \mathrm{m}^{2}$ emitter and $700 \times 350 \mu \mathrm{m}^{2}$ collector is used for further characterization.

\section{Results and discussion}

In Fig. 4, the CIP magnetoresistance of the spin-valve layer is given. A CIP MR of nearly $1 \%$ is observed. The $\mathrm{Si}(100) / \mathrm{Pt} / \mathrm{Py} / \mathrm{Au} / \mathrm{Co}$ structure shows a higher switching field for the Co layer compared to that when an Au is used as capping layer. This is because of the surface oxidation of the Co layer. In Fig. 5 the magnetic field dependence of the collector current of the spin-valve transistor at $220 \mathrm{~K}$ is given. The magnetocurrent is defined as $M C=\left(I_{\mathrm{C}}^{\mathrm{P}}-I_{\mathrm{C}}^{\mathrm{AP}}\right) / I_{\mathrm{C}}^{\mathrm{AP}}$, where $I_{\mathrm{C}}^{\mathrm{P}}$ and

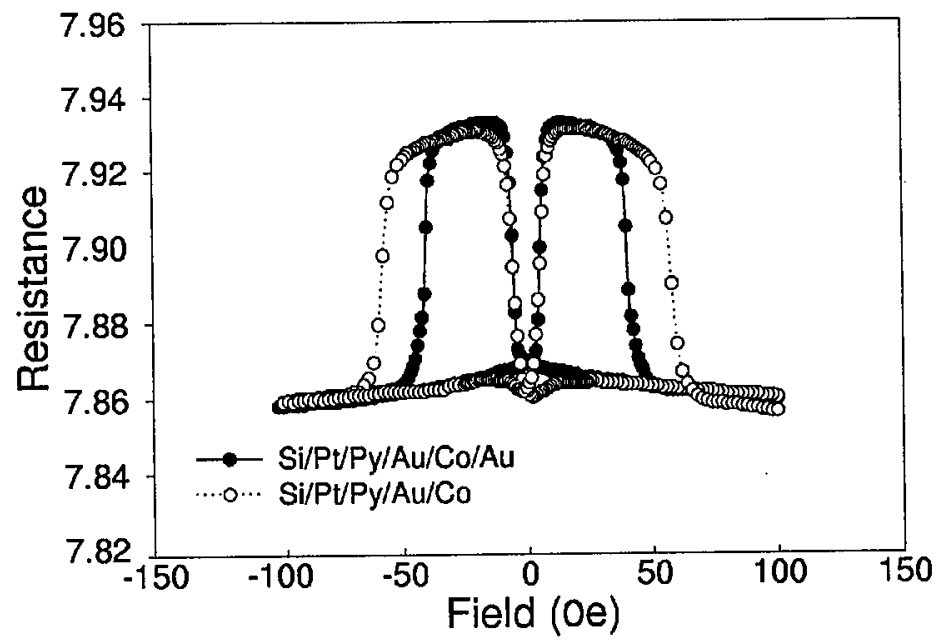

Fig. 4. The CIP magnetoresistance at room temperature for $\mathrm{Si} / \mathrm{Pt}(2 \mathrm{~nm}) / \mathrm{Py}(3 \mathrm{~nm}) /$ $\mathrm{Au}(3.5 \mathrm{~nm}) / \mathrm{Co}(3 \mathrm{~nm})$ and $\mathrm{Si} / \mathrm{Pt}(2 \mathrm{~nm}) / \mathrm{Py}(3 \mathrm{~nm}) / \mathrm{Au}(3.5 \mathrm{~nm}) / \mathrm{Co}(3 \mathrm{~nm}) / \mathrm{Au}(2 \mathrm{~nm})$ sample. 


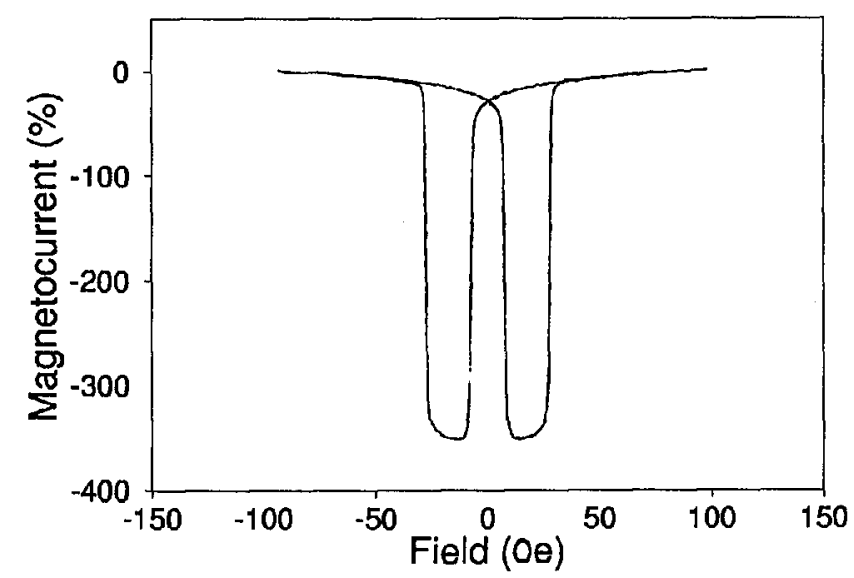

Fig. 5. The magnetic field dependence of magnetocurrent at $220 \mathrm{~K}$ of the spin-valve transistor. $I_{\mathrm{E}}=2 \mathrm{~mA}$ and $V_{\mathrm{BC}}=0$.

$I_{\mathrm{C}}^{\mathrm{AP}}$ are the collector current in the parallel and antiparallel alignment of magnetization, respectively. At $220 \mathrm{~K}$ a magnetocurrent of $\approx 350 \%$ is observed. The collector current depends strongly on scattering of electrons in the base layer. The inelastic scattering due to the defects and electron-electron scattering causes a loss in the energy of these electrons and most of these electrons are not collected as it has smaller energy compared to the collector barrier. An overall reduction in the collector current compared to the emitter current is due to this scattering. An additional scattering of electrons which is associated with the relative orientation of the magnetization of the magnetic layers in the base layer is spin-dependent scattering. When the magnetization of both magnetic layers is parallel the spin-dependent scattering is smaller and we get a collector current of nearly $10 \mathrm{nA}$. When the magnetization is antiparallel the enhancement of spin-dependent scattering lowers the collector current, so the collector current is very sensitive to the relative orientation of magnetization of magnetic layers.

It is known that an imbalance of spin population at the Fermi level constitutes spin-polarized transport in materials. In ferromagnetic metals the density of states available to spin up and spin down electrons is nearly identical but these states are shifted in energy with respect to each other. This results in an unequal filling of the bands which in turn makes the spin up and spin down carriers at the Fermi level to be unequal in number and mobility. This causes a spin-dependent transport for the Fermi electrons across the spin-valve layer and is believed to be the origin for the magnetoresistance in the normal spin-valve configuration. It is also known that when the electrons are passed through all the magnetic layers (CPP mode) the resultant magnetoresistance is higher compared to that of the CIP mode. In a spin-valve transistor the charge carrier is hot electron and has energy nearly $0.85 \mathrm{eV}$ above the Fermi level. Theoretical studies have shown that the band asymmetry for the spin up and spin down electron is high at energies above the Fermi level [11]. Hence in the spin-valve transistor the spin-dependent perpendicular transport of hot electrons as well as the energy and momentum selection of the hot electrons at the collector may be responsible for the large magnetocurrent. 


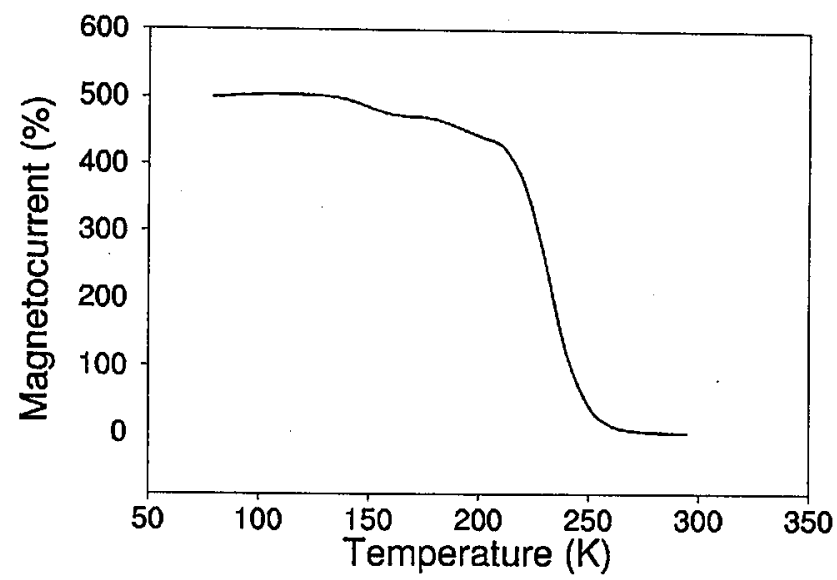

Fig. 6. The temperature dependence of magnetocurrent of the spin-valve transistor. $I_{\mathrm{E}}=2 \mathrm{~mA}$ and $V_{\mathrm{BC}}=0$.

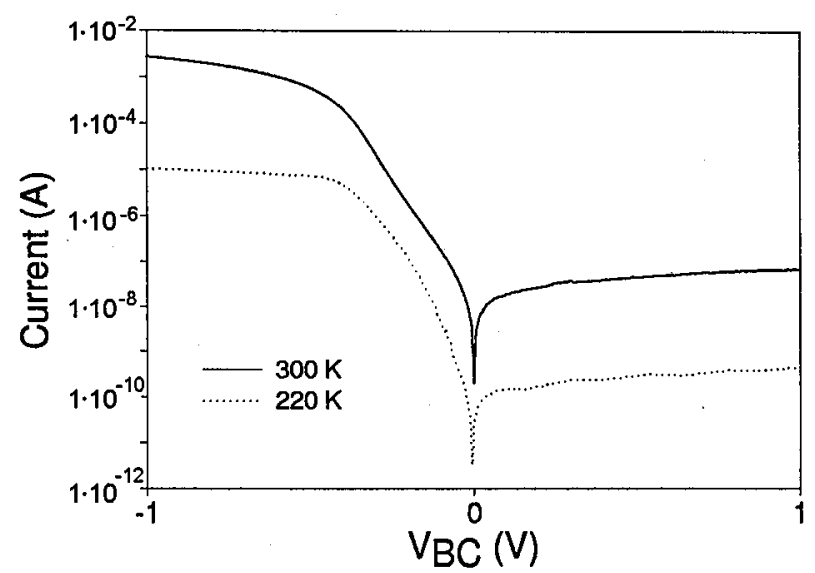

Fig. 7. The $I-V$ characteristics of the collector Schottky diode at room temperature as well as at $220 \mathrm{~K}$.

In Fig. 6 the temperature dependence of the magnetocurrent is given. It is to be noted that $M C \approx 500 \%$ at $80 \mathrm{~K}$. It is found that the magnetocurrent falls rapidly above $220 \mathrm{~K}$ and it has negligible value at room temperature. The collector current at room temperature was found to be very high. In order to understand this the $I-V$ characteristics of the collector diode at room temperature as well as at $220 \mathrm{~K}$ is given in Fig. 7. The leakage current of the collector diode at room temperature is very high and is the reason for the low value of magnetocurrent at room temperature. An improvement in the collector diode characteristic i.e. a reduction in the collector leakage current is expected to improve the performance of the spin-valve transistor at room temperature. 


\section{Acknowledgment}

The authors are thankful to E.G. Keim for TEM studies and R. Jansen, O.M.J. van't Erve, and R. Vlutters for experimental help. Financial assistance from Dutch Technology Foundation (STW) TEL 66.4025 and the European Community [MEL-ARI ESPIRIT 23.307 (SPIDER)] is gratefully acknowledged.

\section{References}

[1] M.N. Baibich, J.M. Broto, A. Fert, F. Nguyeen Van Dan, F. Petroff, P. Etienne, G. Creuzet, A. Friedrich, J. Chazelas, Phys. Rev. Lett. 61; 2472 (1988).

[2] G.A. Prinz, Science 282, 1660 (1998).

[3] S.S.P. Parkin, Z.G. Li, D.J. Smith, Appl. Phys. Lett. 58, 2710 (1991).

[4] M.A.M. Gijs, S.K.J. Lenczowski, J.B. Giesbers, Phys. Rev. Lett. 70, 3343 (1993).

[5] T. Shinjo, H. Yamamoto, J. Phys. Soc. Jpn. 59, 3061 (1990).

[6] H. Yamamoto, Y. Motomura, T. Anno, T. Shinjo, J. Magn. Magn. Mater. 126, 437 (1993).

[7] J.S. Moodera, L.R. Kinder, T.M. Wong, R. Meservey, Phys. Rev. Lett. 74, 3273 (1995).

[8] D.J. Monsma, J.C. Lodder, Th.J.A. Popma, B. Dieny, Phys. Rev. Lett. 74, 5260 (1995).

[9] K. Mizushima, T. Kinno, K. Tanaka, T. Yamauchi, Phys. Rev. B 58, 4660 (1998).

[10] T. Shimatsu, R.H. Mollema, D. Monsma, E.G. Keim, J.C. Lodder, J. Vac. Sci. Technol. A 16, 2125 (1998).

[11] E.Yu. Tsymbal, D.G. Pettifor, J. Appl. Phys. 81, 4579 (1997). 